\title{
Minimally Invasive Techniques in Orthopedic Trauma
}

\author{
M. Thaeter ${ }^{1,2} \cdot$ P. Kobbe ${ }^{1,2} \cdot$ E. Verhaven ${ }^{3} \cdot$ H.-C. Pape ${ }^{1,2}$
}

Published online: 27 October 2016

(C) Springer International Publishing AG 2016

\begin{abstract}
Purpose of Review This review focuses on the evolution of minimally invasive techniques in orthopedic surgery and provides an overview of the evidence-based knowledge of the last decade.

Recent Findings Implants and surgical techniques for minimally invasive osteosynthesis in periarticular, spinal and pelvic fractures underwent rapid improvement. They show adequate healing along with low complication rates and satisfactory outcome. Computer-assisted insertion of implants and navigation offer new possibilities and are particularly advantageous in difficult anatomical regions, such as the pelvis or spine.

Summary The treatment purpose in minimally invasive orthopedic surgery consists of anatomic reconstruction and
\end{abstract}

This article is part of the Topical Collection on Minimally Invasive Surgical Techniques for Trauma

M. Thaeter

mthaeter@ukaachen.de

P. Kobbe

pkobbe@ukaachen.de

E. Verhaven

verhaven.eddy@skynet.be

H.-C. Pape

hpape@ukaachen.de

1 Harald Tscherne Research Laboratory for Orthopaedic Trauma, Aachen University Medical Centre, Pauwelsst. 30, 52074 Aachen, Germany

2 Department of Orthopaedic Trauma, Aachen University Medical Centre, Pauwelsst. 30, 52074 Aachen, Germany

3 Department of Orthopaedic Surgery, St. Nicolaus Hospital, Hufengasse 4, 4700 Eupen, Belgium stabilization of the articular surface, length, axis and rotation with minimal soft-tissue trauma. This review article highlights the surgical evolution and discusses recent advances in techniques and technology implementing minimally invasive approaches as the gold standard of treatment in the near future.

Keywords Minimally invasive surgery $\cdot$ Trauma ·

Orthopedics $\cdot$ Extremity $\cdot$ Spine $\cdot$ Pelvis

\section{Introduction}

After anesthesia and asepsis, the introduction of minimally invasive surgery (MIS) is called the third patient-friendly revolution in surgery. Due to shorter duration of in-hospital treatment and better cosmetic results, arthroscopic surgical techniques have widely replaced open surgery in articular surgery. Implants and surgical techniques for minimally invasive plate osteosynthesis (MIPO) and minimally invasive percutaneous plate osteosynthesis (MIPPO) in periarticular fractures underwent rapid improvement and demonstrate adequate healing along with low complication rates. Computerassisted minimally invasive insertion of implants and navigation offer new possibilities and are particularly advantageous in difficult anatomical regions, such as the pelvis or spine.

Yet, randomized clinical trials are missing to prove the advantages of minimal invasive surgery [1]. Nevertheless, during the past decade, a large number of these high quality randomized controlled trials and meta-analyzes detected significant advantages over open surgery - based in particular on blood loss, soft-tissue trauma, pain-associated parameters, and outcome.

Through continuous development of arthroscopic and minimally invasive surgical techniques, instruments, and implants paired with sophisticated digital navigation technologies, 
minimally invasive trauma surgery will be reaching gold standard of treatment in the near future.

The purpose of this review is to give an overview of the current possibilities and limitations of minimally invasive treatment in trauma surgery.

\section{Extremity}

The majority of injuries in orthopedic surgery are diaphyseal or periarticular fractures of the limb. The concept of "absolute anatomical stability", where each fragment either from the articular or metaphyseal area was supposed to be subject for anatomical reduction and stabilization using rigid implants has been associated with poor long-term biological effects. As a result of large incisions and vast deperiostation, complications such as infection, delays in consolidation, and nonunion occurred [2]. These findings lead to the "biological (bridging) plate osteosynthesis" concept and initiate a remarkable development of new surgical techniques, e.g., indirect or closed reduction and MIPO, and implants, e.g., bridging plates, Limited Contact-Dynamic Compression Plate (LCDCP), or unidirectional and recently multidirectional angular screw stability [3]. A certain (relative) instability degree can be useful for accurate and fast healing. The advantages of ameliorated bone vascularization, callus formation [4] and consolidation, shorter operative time, less blood loss, and decreased infection rate have made these minimally invasive techniques a state-of-the-art treatment. By increased resilience to mechanical stress, implant insertion and removal allow earlier patient rehabilitation [5].

For most diaphyseal fractures, minimally invasive surgical treatment is routinely applied. For acute displaced clavicle shaft fracture, MIPO is an equally effective and safe treatment method when compared to open plating with interfragmentary screw fixation [6] providing better cosmetic results and patient satisfaction. In view of shorter surgical and fluoroscopy time, intramedullary nailing may offer an alternative [7]. Surgical options on the adult humeral shaft fracture include external fixation, open reduction and internal fixation (ORIF), MIPO, and ante-/retrograde intramedullary nailing. MIPOs using the dual approaches show an excellent bony union decreasing the risk of iatrogenic radial nerve palsy compared to $\operatorname{ORIF}[8,9]$. While plating remains an option in some type of fracture, e.g., periprosthetic fractures, medullary nailing after drilling out the medullary cavity is the method of choice in treatment of fractures of the femur shaft. Most nails can be positioned in an anterograde or retrograde direction and allow immediately full-weight bearing $[10,11]$.

The treatment of complex periarticular fractures of the long bones remains particularly challenging. In proximal humeral fractures, a recent multicenter study and a meta-analysis provide evidence that both intramedullary nailing and MIPO through the deltoid-splitting approach are safe and effective in enabling an early return of shoulder function $[12,13]$. The choice of treatment option in distal radius fracture should results from an individual decision mainly based on complexity and stability of the fracture and the patient's age. For stable fractures, functional treatment after closed reduction or percutaneous fixation using the Kapandji technique should be performed. The use of fixed angle intramedullary devices in extra-articular or simple intra-articular distal radius fractures is gaining popularity, limiting soft tissue dissection and tendon irritation while affording adequate stability for early wrist motion. Systematic reviews reveal excellent functional outcomes. However, the mean complication rate of $17.6 \%$ for intramedullary nailing is higher, notably because of the propensity to cause a transient neuritis of the superficial branch of the radial nerve $[14,15]$.

A recent meta-analysis compared two common surgical treatments for intertrochanteric fractures of the proximal femur reveals advantages of the percutaneous compression plate (PCCP) with less need of transfusion, reduced hospital stay, and fewer incidences of implant-related complications compared to intramedullary nail (IMN) fixation [16]. MIPO in distal femur fracture fixation is safe, efficient [17], and associated with less implant failure and nonunion compared to ORIF [18] while its higher risk of rotational malalignment remains despite existing techniques for control of axes and rotation [19•]. While rates of malunion remain still improvable, the advantages of minimally invasive surgery in treatment of distal tibial fractures with its principles of the gentlest possible soft-tissue handling and avoidance of unnecessary dissection become undisputable [20-22]. Through a distal anterolateral approach, concomitant distal fibular fracture can be treated at the same level using MIPO-technique [23].

\section{Spine}

The important role of minimally invasive surgery in spinal fractures is undisputed. Vertebral compression fractures that may be caused by bone-weakening conditions (such as osteoporosis or spine cancer in the elderly) or by minor trauma injury are commonly treated by minimally invasive vertebral augmentation procedures such as vertebroplasty and kyphoplasty. These surgeries show high success rates in terms of improving the patient's pain, early rehabilitation, and fracture stabilization, avoiding more aggressive and larger open spinal fusion surgery [24].

Atlantoaxial instabilities caused by different types of fractures (especially odontoid fractures including nonunion, transverse ligament ruptures, or unstable Jefferson fractures), tumors or infections requiring reduction and stabilization of the atlantoaxial joint underwent a continuous development of surgical techniques and strategies. 
Fig. 1 (left and right) reposition und fixation using $\mathrm{C} 1$ lateral mass and $\mathrm{C} 2$ isthmus-screw fixateur interne described by Harms

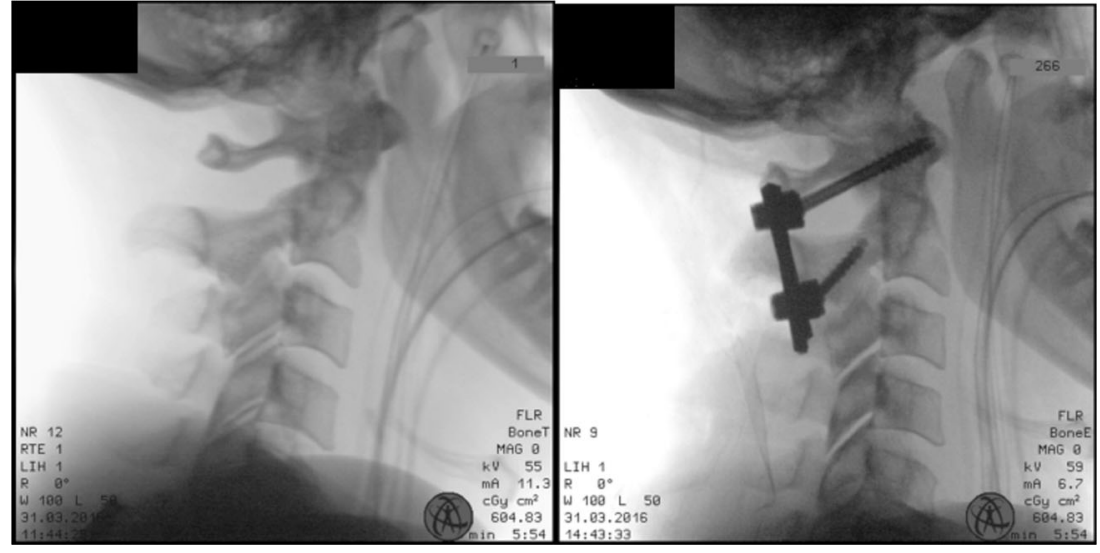

Still remaining a challenging procedure for the surgeon $\mathrm{C} 1$ lateral mass and $\mathrm{C} 2$ pedicle screw combined as a fixateur interne described by Harms using a posterior incision has been widely accepted as it provides sufficient biomechanical stability by offering the possibility of reduction (Fig. 1) and vertebral fusion while minimizing the risk of an injury to the vertebral artery. In case of insufficient screw channel size, isthmal screws can be inserted (Fig. 1, right). Preoperative CT- or MRI-angiography is recommended to assess potential anatomical variations of the artery trajectories. Computernavigated posterior atlantoaxial fixation with the Magerl screw technique is a true minimal invasive option (Fig. 2). While allowing adequate reduction, vertebral fusion however cannot be performed. This technique seems promising for immunocompromised patients (e.g., diabetes and cancer) by reducing the risk of wound infection.

The invasive open dorsal approach for traumatic fractures of the thoracic or lumbar spine (AO type A3/A4, B, and C injuries) is increasingly replaced by the use of percutaneous systems as the development of reduction tools compensates its major drawback: the limited ability to reduce the fracture (Fig. 3). The advantages of percutaneous systems become especially evident in multi- segmental dorsal stabilization: shorter operation time, reduced blood loss and soft-tissue trauma and lower rates of wound infections allow early patient mobilization and shorten the length of hospital stay. There is no difference in the accuracy of pedicle screw positioning and the restoration of sagittal balance as a major goal of spinal fracture treatment. Nevertheless, mid- and long-term functional outcomes seem not to be influenced by a percutaneous dorsal approach [25]. In osteoporotic bone, additional stability can be achieved by cement augmentation. Both pain reduction and less screws loosening have been described to occur [26]. In terms of intense reduction possibilities and spinal cord decompression, the open approaches still remain superior to the percutaneous systems although minimally invasive decompression is also a viable option [27••, 28].

In case of severe destruction of the vertebral bone, patients can benefit from anterior column reconstruction, preventing a progressive loss of reduction by maintaining the physiological load distribution (Fig. 4, center and right). While requiring single-lung ventilation, thoracoscopic techniques replace open thoracotomy and thoracophrenolumbotomy and avoid extensive soft tissue dissection and rib resection (Fig. 4; left) [29••]. The use of computer-aided 3D imaging has proven its value by improving
Fig. 2 (left, center, right) computer-navigated posterior atlantoaxial fixation with the Magerl screw technique

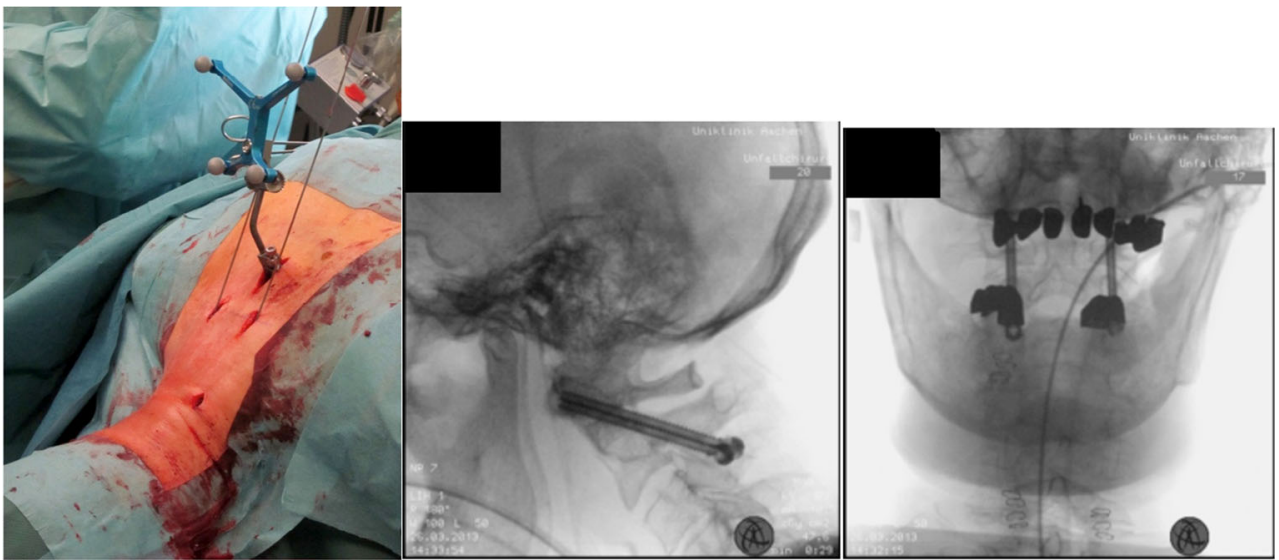




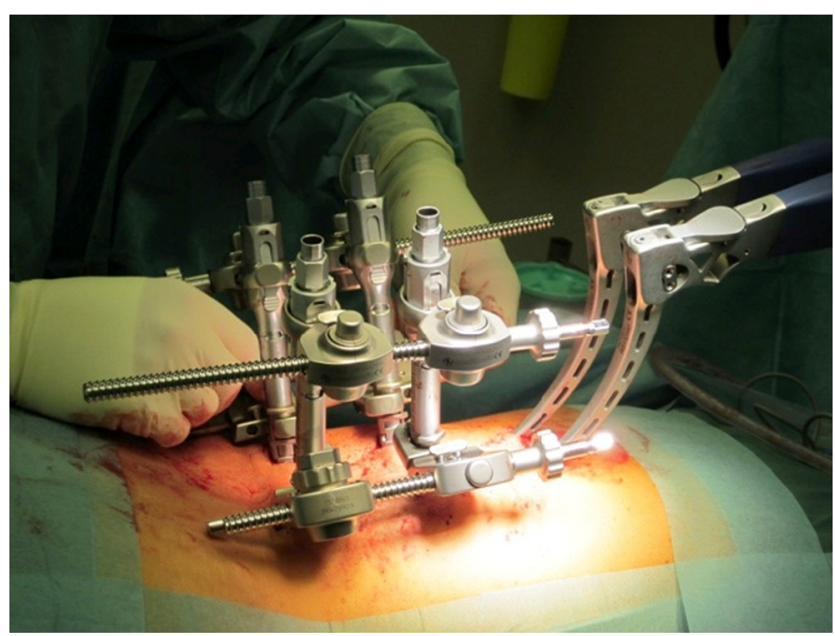

Fig. 3 Reposition tool for minimally invasive dorsal instrumentation

image quality for the anatomical landmarks, especially the complex atlantoaxial region, safety, and accuracy compared to conventional fluoroscopy [30•].

\section{Pelvis}

Pelvic ring injuries represent a therapeutic challenge to the orthopedic surgeon. Surgical stabilization is indicated in unstable injury patterns (with or without radiographic dislocation). Also, it is recommended with failing nonsurgical management. The indication is largely based on fracture classification, associated injuries, and the patient's physiological status. Open reduction and internal fixation is routinely applied but minimally invasive percutaneous plate- or screw fixation $[31,32]$ as well as the anterior fixation of the pelvic ring with addressed compression tools is gaining popularity. The internal use of the anterior fixator (Fig. 5) as a definitive treatment in hemodynamically stable patients provides a novel approach by eliminating the disadvantages of difficult nursing care and pin-infections, especially in obese patients, and allowing the patient to sit upright and even in prone position. In case of

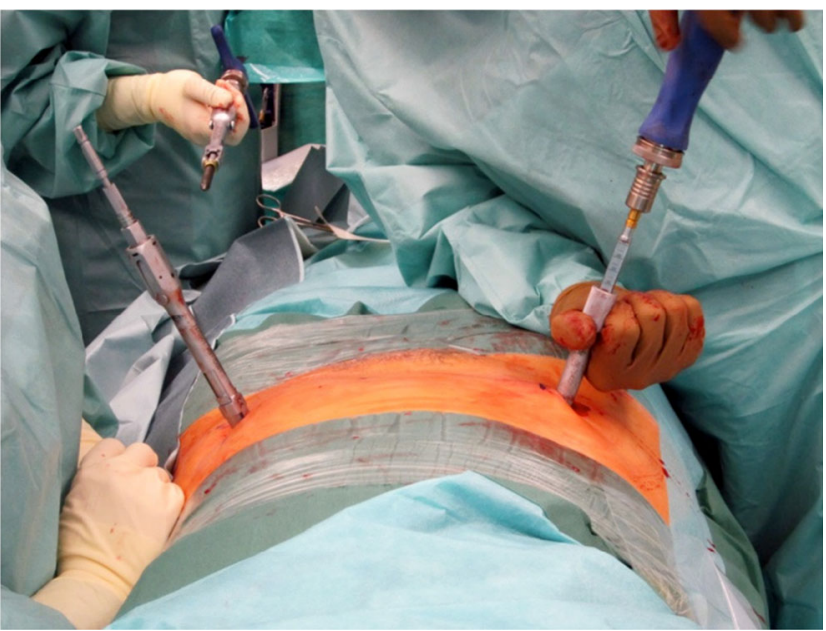

Fig. 5 Screw placement for percutaneous anterior internal pelvic fixator (CD Horizon Longitude, Medtronic, Memphis, TN)

vertical instability (egg APC 3), posterior stability has to be recovered previously by reduction and fixation with either iliosacral screws or posterior plating of spinopelvic fixation $[33,34,35 \bullet \bullet$.

Operative treatment of acetabular fractures remains one of the most challenging sections in trauma surgery. Since minor articular gaps may lead to posttraumatic arthritis, precise reduction is essential in order to avoid poor functional outcome. Open reduction and internal fixation is considered the gold standard of treatment, especially in displaced fractures involving the weight-bearing surface or the posterior wall. For acetabular fractures in the elderly as well as less- or undisplaced fractures in patients aiming for an early return activity, percutaneous techniques with cannulated screws [36] or minimally invasive plate osteosynthesis (MIPO) using preshaped 3D plates [37] are on a rise. Although long-term outcomes are limited, most advanced treatment methods including minimally invasive fixation under computer-assisted navigation [38] and 3D printing, simulating the plate preflex and screw length [39], are promising to improve surgical accuracy and safety and to shorten the operation time.
Fig. 4 (left, center, right) thoracoscopic vertebral body replacement Th12 after minimally invasive dorsal instrumentation

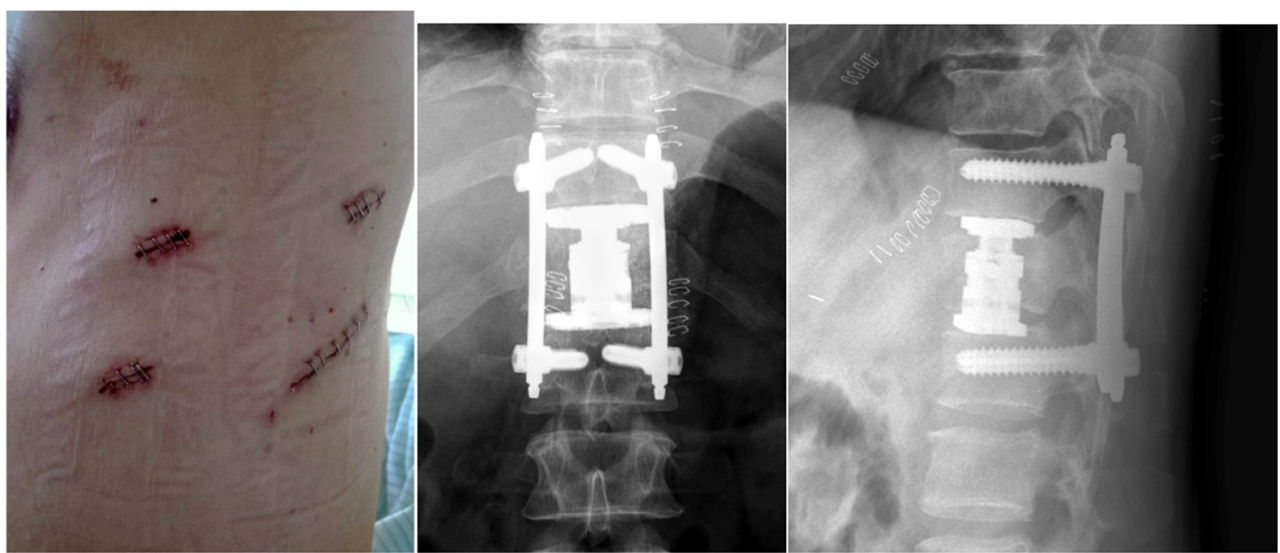




\section{Discussion and Conclusion}

The treatment purpose in minimally invasive orthopedic surgery consists of anatomic reconstruction and stabilization of the articular surface, length, axis, and rotation with minimal soft-tissue trauma. For the metaphyseal-diaphyseal area, this concept is implemented through intramedullary implants or plate osteosynthesis fixated only distally and proximally from the fracture site, bridging the fracture area and leading to biological advantages. Better knowledge in metallurgy and biomechanics paired with advanced instruments, techniques, imaging, and navigation results in improved possibilities for the treatment of truncal and cervical injuries. Combining less invasiveness and stability, this development leads to higher potential for immediate post-operative full weight bearing, which is particularly beneficial to elderly patients [40]. Furthermore, by minimizing additional insults ("second hits"), modern minimal invasive techniques will contribute to the concept of damage control orthopedic surgery for severely injured and polytraumatized patients.

Although demanding, minimally invasive techniques appear to warrant the effort to master the learning curve.

\section{Compliance with Ethical Standards}

Conflict of Interest Drs. Thaeter, Kobbe, Verhaven, and Pape declare no conflicts of interest relevant to this manuscript.

Human and Animal Rights and Informed Consent This article does not contain any studies with human or animal subjects performed by any of the authors.

\section{References}

Papers of particular interest, published recently, have been highlighted as:

- Of importance

•- Of major importance

1. Egol KA. Minimally invasive orthopaedic trauma surgery: a review of the latest techniques. Bull Hosp Joint Dis (New York, NY). 2004;62(1-2):6-12.

2. Baumgaertel F, Buhl M, Rahn BA. Fracture healing in biological plate osteosynthesis. Injury. 1998;29 Suppl 3:C3-6.

3. Sirbu PD, Petreus T, Asaftei R, Berea G, Botez P. Minimally invasive plate osteosynthesis (MIPO) in long bone fractures - biomechanics - design - clinical results. "Gr.T.Popa" University of Medicine and Pharmacy Iasi, Romania. 2011. http://www. intechopen.com/books/biomechanics-in-applications/minimallyinvasive-plate-osteosynthesismipo-in-long-bone-fracturesbiomechanics-design-clinical-res. Accessed July 19, 2016.

4. Xu H, Xue Z, Ding H, Qin H, An Z. Callus formation and mineralization after fracture with different fixation techniques: minimally invasive plate osteosynthesis versus open reduction internal fixation. PLoS One. 2015;10(10), e0140037.
5. Zou T, Li Q, Zhou X, et al. Remove orthopedic fracture implant with minimal invasive surgery is good for the patient's early rehabilitation. Int J Clin Exp Med. 2015;8(12):22377-81.

6. Sohn HS, Kim WJ, Shon MS. Comparison between open plating versus minimally invasive plate osteosynthesis for acute displaced clavicular shaft fractures. Injury. 2015;46(8):1577-84.

7. Zehir S, Zehir R, Sahin E, Calbiyik M. Comparison of novel intramedullary nailing with mini-invasive plating in surgical fixation of displaced midshaft clavicle fractures. Arch Orthop Trauma Surg. 2015;135(3):339-44.

8. Carroll EA, Schweppe M, Langfitt M, Miller AN, Halvorson JJ. Management of humeral shaft fractures. J Am Acad Orthop Surg. 2012;20(7):423-33.

9. Lee T, Yoon J. Newly designed minimally invasive plating of a humerus shaft fracture; a different introduction of the plate. Int Orthod (SICOT). 2016. doi:10.1007/s00264-015-3097-8.

10. Logters T, Windolf J, Flohe S. Fractures of the shaft of the femur. Unfallchirurg. 2009;112(7):635-50. quiz 51.

11. Neumann MV, Sudkamp NP, Strohm PC. Management of femoral shaft fractures. Acta Chir Orthop Traumatol Cechoslov. 2015;82(1):22-32.

12. Falez F, Papalia M, Greco A, et al. Minimally invasive plate osteosynthesis in proximal humeral fractures: one-year results of a prospective multicenter study. Int Orthop. 2016;40(3):579-85.

13. Wang G, Mao Z, Zhang L, et al. Meta-analysis of locking plate versus intramedullary nail for treatment of proximal humeral fractures. J Orthop Surg Res. 2015;10:122.

14. Hardman J, Al-Hadithy N, Hester T, Anakwe R. Systematic review of outcomes following fixed angle intramedullary fixation of distal radius fractures. Int Orthop. 2015;39(12):2381-7.

15. Jordan RW, Saithna A. Defining the role of intramedullary nailing for fractures of the distal radius: a systematic review. Bone Joint J. 2015;97-b(10):1370-6.

16. Shen J, Hu C, Yu S, Huang K, Xie Z. A meta-analysis of percutenous compression plate versus intramedullary nail for treatment of intertrochanteric HIP fractures. Int J Surg (London, England). 2016;29:151-8.

17. Polat A, Kose O, Canbora K, Yanik S, Guler F. Intramedullary nailing versus minimally invasive plate osteosynthesis for distal extra-articular tibial fractures: a prospective randomized clinical trial. J Orthop Sci : Off J Jpn Orthop Assoc. 2015;20(4):695-701.

18. Lill M, Attal R, Rudisch A, Wick MC, Blauth M, Lutz M. Does MIPO of fractures of the distal femur result in more rotational malalignment than ORIF? A retrospective study. Eur J Trauma Emerg Surg. 2015. doi:10.1007/s00068-015-0595-8.

19. Krettek C, Miclau T, Grun O, Schandelmaier P, Tscherne H. Intraoperative control of axes, rotation and length in femoral and tibial fractures. Technical note. Injury. 1998;29 Suppl 3:C29-39. Important and applicable work in the field of minimally invasive surgery.

20. Zhang QX, Gao FQ, Sun W, Wang YT, Yang YR, Li Z. Minimally invasive percutaneous plate osteosynthesis versus open reduction and internal fixation for distal tibial fractures in adults: a meta-analysis. Zhongguo Gu Shang = China J Orthop Traumatol. 2015;28(8):757-62.

21. Dhakar A, Annappa R, Gupta M, Harshwardhan H, Kotian P, Suresh PK. Minimally invasive plate osteosynthesis with locking plates for distal tibia fractures. J Clin Diagn Res : JCDR. 2016;10(3):RC01-4.

22. Vidovic D, Matejcic A, Ivica M, Jurisic D, Elabjer E, Bakota B. Minimally-invasive plate osteosynthesis in distal tibial fractures: results and complications. Injury. 2015;46 Suppl 6:S96-9.

23. Unlu S, Catma MF, Bilgetekin YG, et al. Minimally invasive plate osteosynthesis of distal tibia and fibular fractures through a single distal anterolateral incision. J Foot Ankle Surg : Off Publ Am Coll Foot Ankle Surg. 2015;54(6):1081-4. 
24. Kammerlander C, Neuerburg C, Verlaan JJ, Schmoelz W, Miclau T, Larsson S. The use of augmentation techniques in osteoporotic fracture fixation. Injury. 2016;47 Suppl 2:S36-43.

25. Liu L, Liu GW, Ma C. Comparison of the clinical safety and efficacies of percutaneous pedicle screw fixation and open pedicle screw fixation for thoracolumbar fracture: a meta-analysis. Zhongguo $\mathrm{Gu}$ Shang $=$ China J Orthop Traumatol. 2016;29(3):220-7.

26. Amendola L, Gasbarrini A, Fosco M, et al. Fenestrated pedicle screws for cement-augmented purchase in patients with bone softening: a review of 21 cases. J Orthop Traumatol. 2011;12(4):193-9.

27.• Kobbe P, Pishnamaz M, Lange H, Pape H-C. Minimally invasive dorsal stabilization of traumatic thoracolumbar instabilities. Oper Tech Orthop. 2013;23(1):13-8. Publications from our own fields of expertise in minimally invasive surgery.

28. McAnany SJ, Overley SC, Kim JS, Baird EO, Qureshi SA, Anderson PA. Open versus minimally invasive fixation techniques for thoracolumbar trauma: a meta-analysis. Glob Spine J. 2016;6(2):186-94.

29.• Kobbe P, Pishnamaz M, Pape H-C, Schnake KJ. Thoracoscopic vertebral body replacement in spinal trauma. Oper Tech Orthop. 2013;23(1):19-27. Publications from our own fields of expertise in minimally invasive surgery.

30. Jarvers J-S. Minimally invasive posterior C1/2 screw fixation using C1 lateral mass screws and C2 pedicle screws with 3D C-Armbased navigation. Oper Tech Orthop. 2013;23(1):2-8. Important and applicable work in the field of minimally invasive surgery.

31. Liu Z, Wang K, Zhang K, Zhou J, Zhang Y. Minimally invasive surgery (MIS) of anterior ring fracture combined with pubic symphysis separation. Med Sci Monit : Int Med J Exp Clin Res. 2014;20:1913-7.
32. Arduini M, Saturnino L, Piperno A, Iundusi R, Tarantino U. Fragility fractures of the pelvis: treatment and preliminary results. Aging Clin Exp Res. 2015;27 Suppl 1:S61-7.

33. Zhou KH, Luo CF, Chen N, Hu CF, Pan FG. Minimally invasive surgery under fluoro-navigation for anterior pelvic ring fractures. Indian J Orthop. 2016;50(3):250-5.

34. Halawi MJ. Pelvic ring injuries: surgical management and longterm outcomes. J Clin Orthop Trauma. 2016;7(1):1-6.

35.• Sellei RM, Dienstknecht T, Brücken D, Pfeifer R, Pape H-C. Percutaneous anterior internal pelvic fixator. Oper Tech Orthop. 2013;23(1):33-7. Publications from our own fields of expertise in minimally invasive surgery.

36. Bozzio AE, Wydra FB, Mitchell JJ, Ackerson RM, Mauffrey C. Percutaneous fixation of anterior and posterior column acetabular fractures. Orthopedics. 2014;37(10):675-8.

37. Zhuang Y, Cao S, Lin Y, Li R, Wang G, Wang Y. Minimally invasive plate osteosynthesis of acetabular anterior column fractures using the two-incision minimally invasive approach and a preshaped three dimension plate. Int Orthop. 2016;40(10):2157-62.

38. Xiang Z, Wei D, Liu M, et al. Minimally invasive fixation under computer-assisted navigation for treatment of periacetabular fractures, anterior and posterior pelvic ring fractures. Zhongguo Xiu Fu Chong Jian Wai KE Za Zhi = Zhongguo Xiufu Chongjian Waike Zazhi = Chin J Reparative and Reconstr Surg. 2015;29(1):10-3.

39. Zeng CJ, Tan XY, Huang HJ, et al. Clincial effect of 3D printingassisted minimal invasive surgery through a small incision lateral to the rectus abdominis for pelvic fracture. Nan Fang Yi Ke Da Xue Xue Bao = J South Med Univ. 2016;36(2):220-5.

40. Ehlinger M, Scheibling B, Rahme M, et al. Minimally invasive surgery with locking plate for periprosthetic femoral fractures: technical note. Int Orthop. 2015;39(10):1921-6. 